\title{
Dynamical analysis of an epidemic model with saturated incidence rate and vaccination
}

\author{
A. W. Ogunsola ${ }^{1 *}$, O. Adebimpe ${ }^{2}$, B. A. Popoola ${ }^{3}$ \\ ${ }^{1}$ Department of Pure and Applied Mathematics, Ladoke Akintola University of Technology, Ogbomoso, Nigeria \\ ${ }^{2}$ Department of Physical Sciences, Landmark University, Omuaran, Nigeria \\ ${ }^{3}$ Department of Mathematics, Federal College of Education, Osiele, Nigeria \\ *Corresponding author E-mail: amossolawale@yahoo.com
}

Copyright $\odot 2014$ A. W. Ogunsola et al. This is an open access article distributed under the Creative Commons Attribution License, which permits unrestricted use, distribution, and reproduction in any medium, provided the original work is properly cited.

\begin{abstract}
An epidemic model with saturated incidence rate and vaccination is investigated. The model exhibits two equilibria namely disease-free and endemic equilibria. It is shown that if the basic reproduction number $\left(R_{0}\right)$ is less than unity, the disease-free equilibrium is locally asymptotically stable and in such case, the endemic equilibrium does not exist. Also, it is shown that if $R_{0}>1$, the disease is persistent and the unique endemic equilibrium of the system with saturation incidence is locally asymptotically stable. Lyapunov function and Dulac's criterion plus Poincare-Bendixson theorem are applied to prove the global stability of the disease-free and endemic equilibria respectively. The effect of vaccine in the model is critically looked into.
\end{abstract}

Keywords: Basic Reproduction Number, Dulac's Criterion, Epidemic Model, Lyapunov Function, Poincare-Bendixson Theorem, Vaccination.

\section{Introduction}

Vaccinating susceptible against disease infections is an effective measure to control and prevent the spread of the infection. Kribs-Zaleta and Velasco Hernadez [1] investigated an SIS model with vaccination, standard incidence and no disease-induced diseases. Arino et al. [2] formulated an SIRS epidemic model with vaccination, standard incidence and no disease-induced deaths. Li et al. [3] studied an SIS model with vaccination, standard incidence and diseaseinduced deaths while Brauer [4] investigated an SIS model with vaccination, general incidence and no disease-induced death. $\mathrm{Li}$ and $\mathrm{Ma}$ [5], [6] also analyzed global behaviour of simple SIS vaccination epidemic models under the condition that the vaccine is perfectly efficient.

Adebimpe [7] investigated a SEIV epidemic model with saturated incidence rate that incorporates polynomial information on current and past states of the disease. He showed that if the basic reproduction number $R_{0}<1$, the Disease-Free Equilibrium (DFE) is locally asymptotically stable and by the use of Lyapunov function, DFE is globally asymptotically stable and in such a case, the Endemic Equilibrium (EE) is unstable.

Adebimpe et al. [8] investigated the global stability of a SEIR epidemic model with saturating incidence rate. They identified a threshold $R_{0}$ which determines the outcome of the disease. They used Dulac's criterion plus PoincareBendixson theorem and Lyapunov functions are used to prove the global stability of the disease-free and endemic equilibrium respectively.

Ullah et al. [9] investigated an epidemic model with a vaccination program. They determined the vaccine-induced reproduction number $R_{0}(\mathrm{k})$ and discussed the impact of vaccination in reducing $R_{0}(\mathrm{k})$. Islam et al. [10] constructed a new deterministic model and used to analyze the effect of a preventive vaccine on the transmission dynamics of an infectious disease. 
In this paper, we extend the work done by Islam et al. [10] to incorporate saturated incidence rate of the form $\frac{\beta S I}{1+m_{1} S+m_{2} I}$ as below:

$\frac{d S}{d t}=\delta-\frac{\beta S I}{1+\alpha_{1} S+\alpha_{2} I}-\gamma S-\mu S$

$\frac{d V}{d I}=\gamma S-\mu N-c \beta V I$

$\frac{d I}{d t}=c \beta V I-\frac{\beta S I}{1+\alpha_{1} S+\alpha_{2} I}-\mu I$

Where $\mathrm{S}(\mathrm{t}), \mathrm{V}(\mathrm{t})$ and $\mathrm{I}(\mathrm{t})$ denote the number of the susceptible individuals, vaccinated individuals and recovered individuals respectively. All of the parameters are positive and have the following meaning: $\pi$ is the recruitment of individuals (assumed susceptible) into the population, $\mu$ is the natural death rate, $\gamma$ is the rate of vaccination of the susceptible, $\beta$ is the effective contact rate. Since the vaccine only provides partial protection to the infection, vaccinated individual may still become infected but at the lower rate $c \beta$ than fully susceptible individuals. Here $0<<1$ and $1-\mathrm{C}$ describes vaccine efficacy, when $c=0$, the vaccine is perfectly effective and has no effect on the immunity of vaccinated individuals at all when $\mathrm{c}=1$. $\alpha_{1}$ and $\alpha_{2}$ Are the parameters that measure the effects of sociological, psychological or other mechanisms?

\section{Local stability of the disease-free equilibrium}

The model (1) has a disease-free equilibrium given by $P_{0}=\left(\frac{\delta}{\mu+\gamma}, 0, \frac{\gamma \delta}{\mu(\mu+\gamma)}\right)$. It is obvious that $P_{0}$ attracts the region (stable manifold of $P_{0}$ )

$P_{0}=\{(S, I, V) \in \varphi: \quad I=0\}$

The stability of this equilibrium $P_{0}$ will be investigated using the linearization method governed by the basic reproduction number, $R_{0}$.

Now, by linearizing the system (1) about the point $P_{0}$, we have the following:

Let $S=x+S_{0}, V=y+V_{0}, I=I$

$\frac{d x}{d t}=-\frac{\beta S_{0} I}{1+\alpha_{1} S_{0}}-(\gamma+\mu) x+$ higherorderterms

$\frac{d y}{d I}=\gamma x-\mu y-c \beta V_{0} I+$ higherorderterms

$\frac{d I}{d t}=c \beta V_{0} I+\frac{\beta S_{0} I}{1+\alpha_{1} S_{0}}-\mu I+$ higherorderterms

The Jacobian matrix is

$$
\begin{aligned}
& \left(\begin{array}{l}
\frac{d x}{d t} \\
\frac{d I}{d t} \\
\frac{d y}{d t}
\end{array}\right)=\left(\begin{array}{ccc}
-(\gamma+\mu) & -\frac{\beta S_{0}}{1+\alpha_{1} S_{0}} & 0 \\
0 & c \beta V_{0}+\frac{\beta S_{0}}{1+\alpha_{1} S_{0}}-\mu & 0 \\
\gamma & -c \beta V_{0} & -\mu
\end{array}\right)\left(\begin{array}{l}
x \\
I \\
y
\end{array}\right)+\text { higher order terms } \\
& (\gamma+\mu+\lambda)\left[\left(c \beta V_{0}+\frac{\beta S_{0}}{1+\alpha_{1} S_{0}}-\mu-\lambda\right)(\mu+\lambda)\right]=0 \\
& \lambda_{1}=-(\gamma+\mu), \lambda_{2}=c \beta V_{0}+\frac{\beta S_{0}}{1+\alpha_{1} S_{0}}-\mu, \lambda_{3}=-\mu
\end{aligned}
$$


Since all parameters are assumed positive, so it follows that $\lambda_{1}<0$ and $\lambda_{3}<0$. So, the disease-free equilibrium $P_{0}$ is locally asymptotically stable if and only if $\lambda_{2}=c \beta V_{0}+\frac{\beta S_{0}}{1+\alpha_{1} S_{0}}-\mu<0$.

Let $R=\frac{\beta \delta}{\mu(\mu+\gamma+\alpha \delta)}+\frac{c \beta \gamma \delta}{\mu^{2}(\mu+\gamma)}$

Lemma: The disease-free equilibrium $P_{0}$, of the model (1) is locally asymptotically stable $(C A S)$ if $R_{0}<1$, and unstable if $R_{0}>1$.

The quality $R_{0}$ refers to the average number of secondary cases generated by a single infectious individual in a completely susceptible population. Since $\lambda_{1}, \lambda_{3}$ are negative, if $R_{0}<1, \lambda_{2}<0$. Therefore, the disease-free equilibrium is locally asymptotically stable

\section{Local stability of the endemic equilibrium}

Theorem 1: If the endemic equilibrium $P_{*}\left(S_{*}, I *, V *\right)$ of system ( ) exists, then it is locally asymptotically stable

Proof: According to Theorem (1), the endemic equilibrium $P *(S *, I *, V *)$ exists if and only if $R_{0}>1$. The Jacobian matrix of the system (1) is

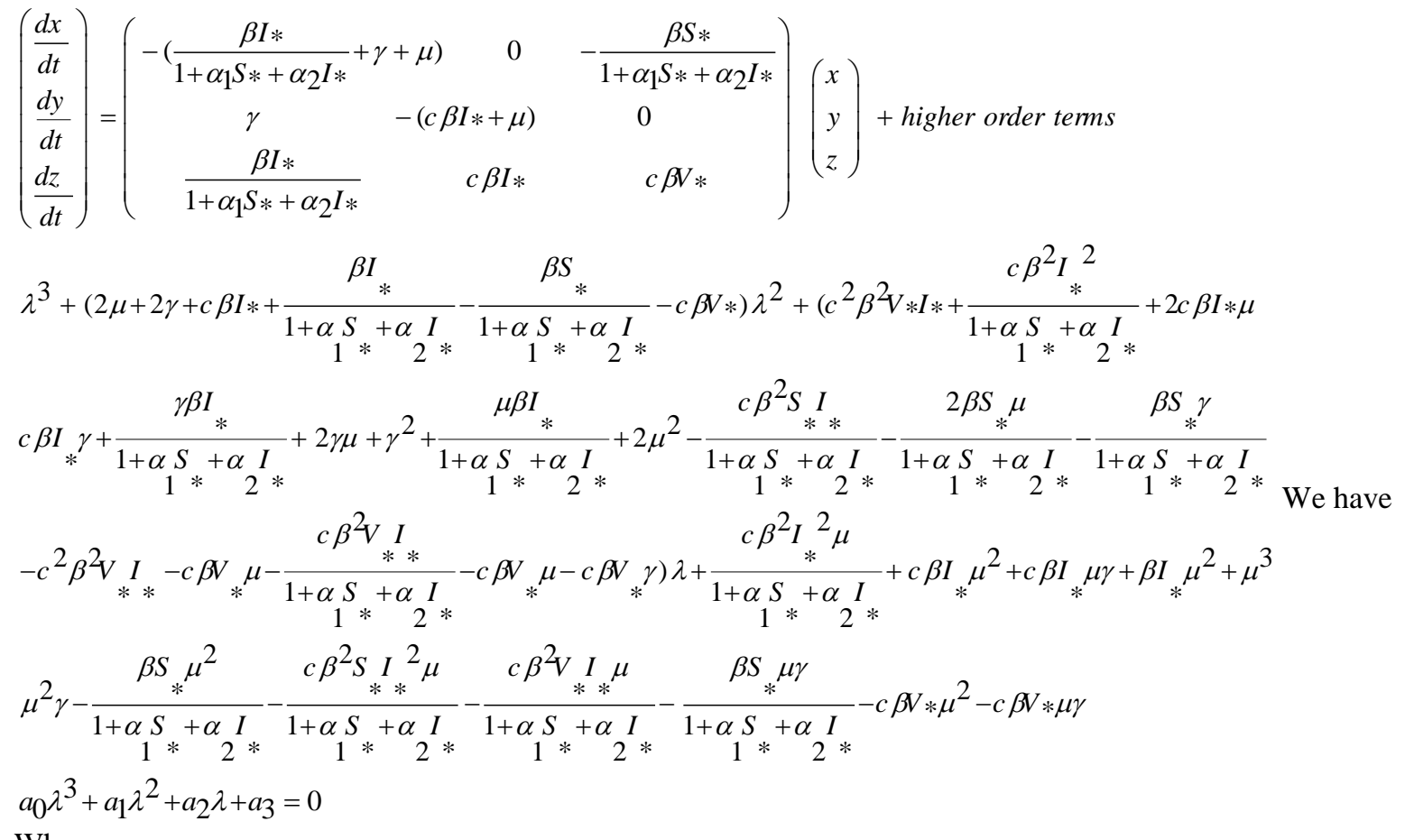

Where

$$
\begin{aligned}
& a_{0}=1, a_{1}=2 \mu+2 \gamma+c \beta I *+\frac{\beta I *}{1+\alpha_{1} S *+\alpha_{2} I *}-\frac{\beta S *}{1+\alpha_{1} S *+\alpha_{2} I *}-c \beta V *, a_{2}= \\
& a_{2}=c^{2} \beta^{2} V * I *+\frac{c \beta^{2} I *{ }^{2}}{1+\alpha_{1} S *+\alpha_{2} I *}+2 c \beta I * \mu c \beta I * \gamma+\frac{\gamma \beta I *}{1+\alpha_{1} S *+\alpha_{2} I *}+2 \gamma \mu+\gamma^{2}+\frac{\mu \beta I *}{1+\alpha_{1} S *+\alpha_{2} I *}+2 \mu^{2}-\frac{c \beta^{2} S * I *}{1+\alpha_{1} S *+\alpha_{2} I *} \\
& -\frac{2 \beta S * \mu}{1+\alpha_{1} S *+\alpha_{2} I *}-\frac{\beta S * \gamma}{1+\alpha_{1} S *+\alpha_{2} I *}-c^{2} \beta^{2} V * I *-c \beta V * \mu-\frac{c \beta^{2} V * I *}{1+\alpha_{1} S *+\alpha_{2} I *}-c \beta V * \mu-c \beta V * \gamma
\end{aligned}
$$




$$
\begin{aligned}
& a_{3}=\frac{c \beta^{2} I *{ }^{2} \mu}{1+\alpha_{1} S *+\alpha_{2} I *}+c \beta I * \mu^{2}+c \beta I * \mu \gamma+\beta I * \mu^{2}+\mu^{3} \mu^{2} \gamma-\frac{\beta S * \mu^{2}}{1+\alpha_{1} S *+\alpha_{2} I *}-\frac{c \beta^{2} S * I *{ }^{2} \mu}{1+\alpha_{1} S *+\alpha_{2} I *}-\frac{c \beta^{2} V * I * \mu}{1+\alpha_{1} S *+\alpha_{2} I *} \text { If } a_{1} a_{2}>a_{0} a_{3}, \text { by } \\
& -\frac{\beta S * \mu \gamma}{1+\alpha_{1} S *+\alpha_{2} I *}-c \beta V * \mu^{2}-c \beta V * \mu \gamma
\end{aligned}
$$

Routh Hurwitz criterion, the endemic equilibrium is locally asymptotically stable.

\section{Global stability of disease-free equilibrium}

In order to prove the global stability of the endemic equilibrium $\mathrm{P} *$ of the equation (1) we apply Dulac's criterion plus Poincare-Bendixson Theorem.

Theorem 2: (Dulac's Criterion)

Consider the following general nonlinear autonomous system of de $x(t)=f(x), x \in E$

Let $f=C^{1}(E)$ where $\mathrm{E}$ is a simple connected region in $\mathrm{R}^{2}$. If the exists a function it $H \in C^{1}(E)$ such that $\nabla$.(Hf ) is not identically zero and does not change sign in E, the system (*) has no close orbit lying entirely in E. if A is an annular region contained in $\mathrm{E}$ on which $\nabla .(H f)$ does not change sign, then there is at most one limit cycle of the system (*) in A.

Theorem 3:(The Poincare-Bendixson Theorem): Suppose that $f \in C^{1}(E)$ where $E$ is an open subset of $R^{n}$ and that the system (*) has a rejecting $\Gamma$ contained in a compact subset $f$ of $E$. assume that the system (*) has only one unique equilibrium point $x_{0}$ in $f$, then one of the following possibilities holds.

a) $\quad \mathrm{w}(\Gamma)$ is the equilibrium point $\mathrm{x}_{0}$

b) $\mathrm{w}(\Gamma)$ is a periodic orbit

c) $\quad \mathrm{w}(\Gamma)$ is a graphic

Theorem 4: Let $P_{*}$ be the unique positive equilibrium point of the system (1). If $R_{0}>1$, then $P_{*}$ of the system (*) is globally asymptotically stable.

Proof: We use Dulac's criterion plus Poincare'-Bendixson Theorem to analyze the system (1). Consider.

$H(S, V, I)=\frac{1}{S V I}$

Where $\mathrm{S}>0, \mathrm{~V}>0, \mathrm{I}>0$. Then,

$$
\begin{aligned}
& \nabla .(H f)=\frac{\partial}{\partial S}\left(H . f_{1}\right)+\frac{\partial}{\partial V}\left(H . f_{2}\right)+\frac{\partial}{\partial I}\left(H . f_{3}\right) \\
& =\frac{\partial}{\partial S}\left[\left(\frac{1}{S V I}\left(\delta-\frac{\beta S I}{1+\alpha_{1} S+\alpha_{2} I}-\gamma S-\mu S\right)+\frac{\partial}{\partial V}\left[\left(\frac{1}{S V I}(\gamma S-\mu V-c \beta V I)+\frac{\partial}{\partial I}\left[\left(\frac{1}{S V I}\left(c \beta V I+\frac{\beta S I}{1+\alpha_{1} S+\alpha_{2} I}-\gamma I\right)\right]\right.\right.\right.\right.\right. \\
& =-\frac{\delta}{S^{2} V I}+\frac{\beta \alpha_{1}}{\left(1+\alpha_{1} S+\alpha_{2} I\right)^{2}}-\frac{\gamma}{V^{2} I}-\frac{\beta \alpha_{2}}{\left(1+\alpha_{1} S+\alpha_{2} I\right)^{2}}
\end{aligned}
$$

If $\alpha_{1}=\alpha_{2}, \nabla$. (Hf $)<0$. Hence, by the Dulac's criterion, there is no closed orbit in the first quadrant. Therefore, the endemic equilibrium is globally asymptotically stable.

\section{Numerical simulations}

In this section, we perform numerical calculations to support our theoretical analysis of this paper. Below are the graphs emanated from the numerical computations?

\section{Discussion of results}

Simulation was carried out with different values of the parameters and stability analysis and values of the threshold were obtained.

From figures 1-4 above, $R_{0}<1$ and different values of $\mathrm{c}$ were used. It was discovered that as $\mathrm{c}$ increases, the more stability the models become. This means that the vaccine has a lot to do with the eradication of the disease.

From figure 5, we have $R_{0}>1$ and different values were used and it was discovered that there is endemic and that means that disease may break out and become endemic.

We established that when $R_{0}<1$, the disease may die out at the long run and when $R_{0}>1$, there may be endemic. 


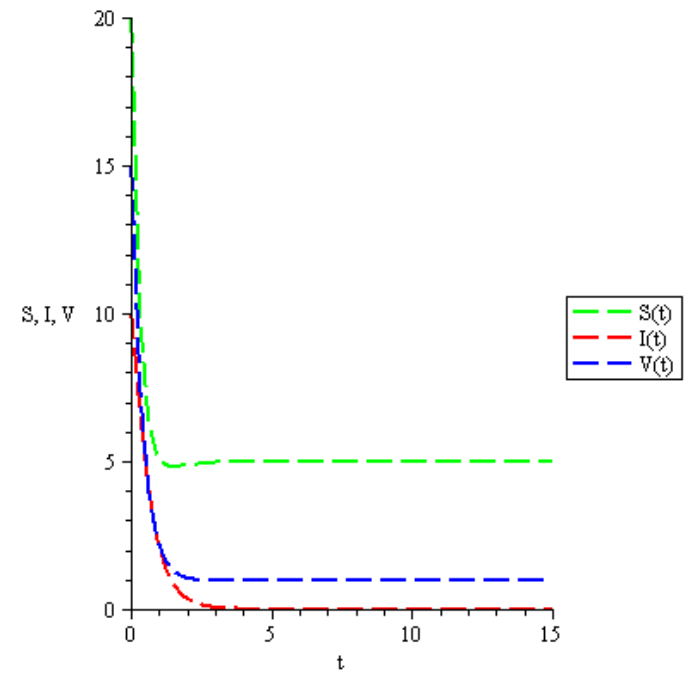

Fig. 1: Graph Of S (T), I (T) And V (T) Against Time (T) When $\delta=15, \beta=0.5, \alpha_{1}=0.5, \alpha_{2}=0.3, \gamma=0.5, \mu=2.5, c=0.1$ and $R_{0}<1$

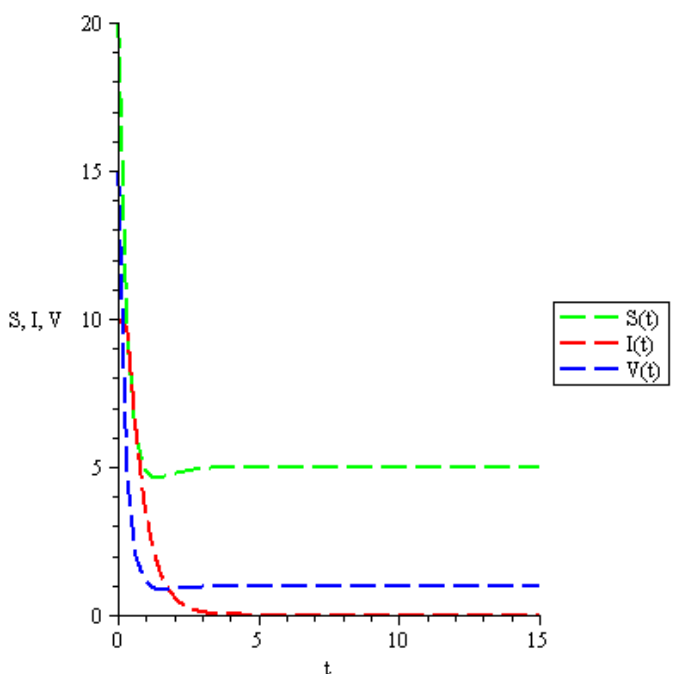

Fig. 2: Graph of S (T), I (T) and V (T) Against Time (T) When $\delta=15, \beta=0.5, \alpha_{1}=0.5, \alpha_{2}=0.3, \gamma=0.5, \quad \mu=2.5, c=0.4$ and $R_{0}<1$

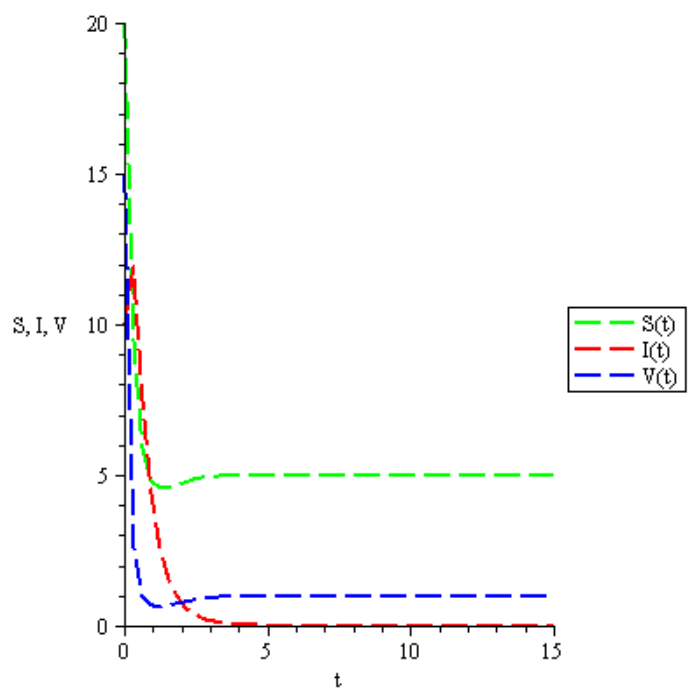

Fig. 3: Graph of S (T), I (T) And V (T) Against Time (T) When $\delta=15, \beta=0.5, \alpha_{1}=0.5, \alpha_{2}=0.3, \gamma=0.5, \mu=2.5, c=0.7$ and $R_{0}<1$ 


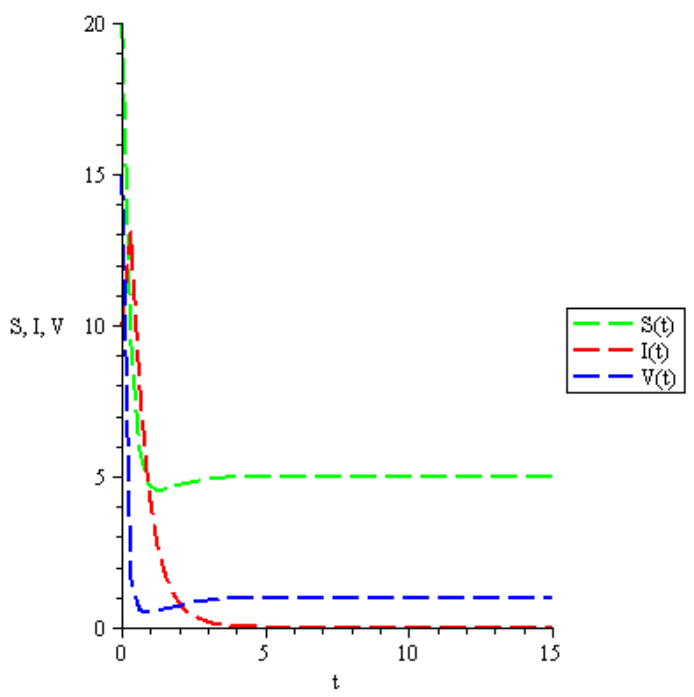

Fig. 4: Graph of S (T), I (T) and V (T) Against Time (T) when $\delta=15, \beta=0.5, \alpha_{1}=0.5, \alpha_{2}=0.3, \gamma=0.5, \mu=2.5, c=0.95$ and $R_{0}<1$

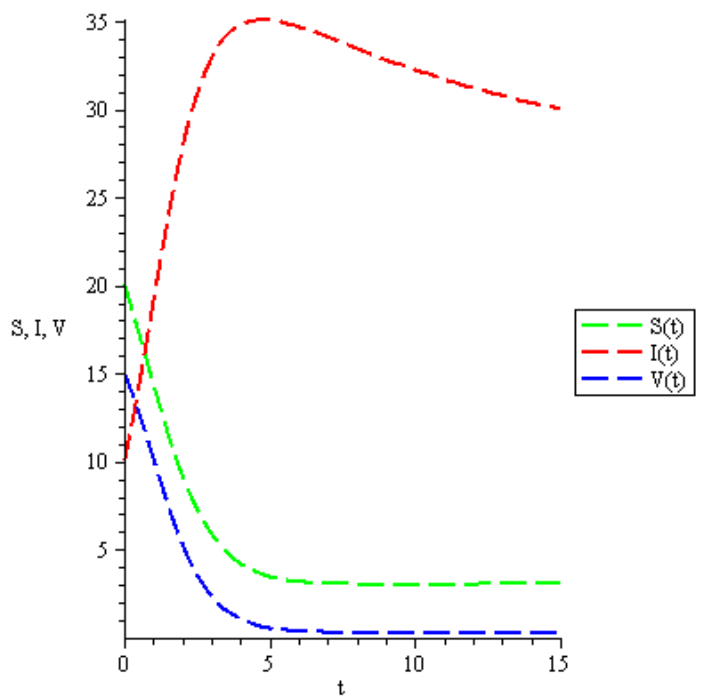

Fig. 5: Graph of S (T), I (T) and V (T) Against Time (T) when $\delta=15, \beta=0.3, \alpha_{1}=0.5, \alpha_{2}=0.3, \gamma=0.1, \quad \mu=0.1, c=0.1$ and $R_{0}>1$

\section{Conclusion}

In this paper, an SVI deterministic model with saturating incidence rate is investigated. Some of the main findings of this study are:

i) The model has a globally-asymptotically stable disease-free equilibrium whenever the associated reproduction number is less than unity;

ii) The model has a unique endemic equilibrium under certain conditions. Under these conditions, the endemic equilibrium is locally-asymptotically stable whenever the associated reproduction number exceeds unity. The endemic equilibrium is shown to be globally-asymptotically stable using Dulac's criterion plus Poincare's Bendixson theorem.

iii) Using Dulac's criterion plus Poincare's -Bendixson theorem and $\alpha_{1}=\alpha_{2}$, the model is globally asymptotically stable.

iv) Numerical Simulations illustrate that the parameter 1-c which describes the vaccine efficacy has a lot to do in disease eradication. As c increases when $R_{0}<1$, there is stability.

This study shows that the disease being considered can be eliminate from the population whenever the basic reproduction number $R_{0}$ is less than unity. The disease persists in the community whenever the basic reproduction number $R_{0}$ exceeds unity. 


\section{References}

[1] C. M.Kribs-Zaleta, J. X.Velasco-Hernadez, A simple vaccination model with multiple endemic states. Math Biosci 164 (2000) 183-201. http://dx.doi.org/10.1016/S0025-5564 (00)00003-1.

[2] J.Arino, C. C.McCluskey, P.van den Driessche, Global results for an epidemic model with vaccination that exhibits backward bifurcation. SIAM J. Appl. Math. Vol.64 No.1 (2003) pp. 260-276. http://dx.doi.org/10.1137/S0036139902413829.

[3] J. Li, Z. Ma, Z., Y. Zhou, Global Analysis of SIS epidemic model with a simple vaccination and multiple endemic equilibrium. Acta Mathematica Scientia B (1): (2006) 83-93.

[4] F. Brauer, Backward bifurcations in simple vaccination models, J. Math. Anl. Appl. 298, (2004) pp. 418-431. http://dx.doi.org/10.1016/j.jmaa.2004.05.045.

[5] J. Li, Z. Ma, Qualitative analyses of SIS epidemic model with vaccination and varying total population size. Math. Comp. Model 35, (2002) 1235-1243. http://dx.doi.org/10.1016/S0895-7177 (02)00082-1.

[6] J. Li, Z. Ma, Global Analysis of SIS epidemic models with variable total population size. Math Comput Model 39 (2004) 1231-1242. http://dx.doi.org/10.1016/j.mcm.2004.06.004.

[7] O. Adebimpe, Stability Analysis of a SEIV Epidemic Model with Saturated incidence Rate, British Journal of Mathematics and Computer Science 4(23): (2014), pp. 3358-3368 http://dx.doi.org/10.9734/BJMCS/2014/2758.

[8] O. Adebimpe, B. O. Moses, O. J. Okoro, Global Stability Analysis of a SEIR Epidemic Model with Saturated Incidence Rate, International Journal of Mathematical Sciences, vol.34, Issue 1, (2014) 1504-1512.

[9] R. Ullah, G. Zahman,S. Islam, I. Ahmad, Dynamical features and vaccination Strategies in an SEIR epidemic model, Research Journal of Recent Sciences, vol.2(10), (2013),pp. 48-56

[10] Md. Saiful Islam, Md. Asaduzzaman, Md. Nazrul Islam Mondal, Stability Analysis of DFE of an Epidemic Model in the presence of a Preventive Vaccine, IOSR Journal of Mathematics, volume 3, issue 2, (2012) pp. 25-31. 